\title{
Descending Thoracic Aortic Aneurysm Rupture Treated with Thoracic Endovascular Aortic Repair in a Patient with Peripheral Artery Disease
}

\author{
Ovidiu Stiru, MD, PhD, Roxana Carmen Geana, MD, Platon Pavel, MD, Marian Croitoru, MD, \\ Cristian Boros, MD, Ion Iovu, MD, V. A. Iliescu, MD, PhD \\ Emergency Institute for Cardiovascular Diseases Prof. Dr. C.C. Iliescu, Bucharest, Romania
}

\section{ABSTRACT}

Descending thoracic aortic aneurysm rupture is a lifethreatening disease associated with high rates of morbidity and mortality. Treatment in these cases is a surgical emergency. Less invasive therapies for the treatment of this pathology have been developed over time. For descending thoracic aneurysm rupture, endovascular stent grafting is less invasive, life-saving, and a unique alternative to open repair. However, this approach is subject to anatomical and logistic limitations.

The purpose of the present study is to report a case of an emergency endovascular repair for a ruptured thoracic aortic aneurysm in a patient with peripheral arterial disease, and to discuss some important issues related to this approach. Severe calcifications were discovered in this patient on both iliac arteries, and the remaining circulated lumen was less than 2 to $3 \mathrm{~mm}$. Unfortunately, only catheter insertion into the left iliac artery for angiography was able to determine the development of a dissection in the arterial wall. We decided to dilate both iliac arteries with partial stabilization of the dissection on the right iliac artery which allowed us to successfully continue the endovascular procedure. After 24 hours, the patient experienced right limb ischemia, and revascularization of the affected limb was achieved by performing a right axillofemoral bypass.

\section{INTRODUCTION}

An aortic aneurysm is a localized arterial dilatation to more than $50 \%$ of the normal aortic diameter [Hager 2002]. Descending aortic aneurysms are considered to represent approximately $10 \%$ of all the thoracic aneurysms [Hager 2002]. The incidence of rupture for aortic thoracic aneurysms is $5 / 100,000$, and $30 \%$ of these involve the descending thoracic aorta [Johansson 1995]. It is a life-threatening disease associated with high rates of morbidity and mortality of up to $97 \%$ [Johansson 1995]. Initially, medial degeneration occurs as an adaptive response to wall stress in the dilated segment of the aorta. Aneurysm development per Laplace's law increases parietal tension, causing further

Received August 20, 2017; received in revised form fanuary 23, 2018; accepted February 9, 2018.

Correspondence: Ovidiu Stiru, MD, PbD, Emergency Institute for Cardiovascular Diseases "Prof. Dr. C.C. Iliescu," Sos. Fundeni 258, sect. 2. Bucharest, Romania, +4072220728 (e-mail: ovidiu_stiru@yahoo.com).. expansion of the aneurysmal segment. Thus, if the aortic wall's resistance limit is exceeded, the aneurysm will break. Treatment in these cases is a surgical emergency.

In 1951, Lam and Aram reported the first resection of a descending thoracic aneurysm with allograft replacement [Lam 1951]. Usually, Dacron grafts (first introduced by DeBakey) are used to replace aortic aneurysmal segments. Less invasive therapies for the treatment of descending thoracic aortic aneurysms have been developed over time. Dake et al reported the first thoracic endovascular aortic repair (TEVAR) in 1994 [Dake 1998]. In 2005, the US Food and Drug Administration (FDA) approved for use three thoracic aortic stent grafts: the GORE TAG graft (W.L. Gore and Associates), the Talent Thoracic endograft (Medtronic), and the Cook TX2 endograft (Cook).

In time, several successful next-generation reiterations of all of these devices have also gained approval. For descending thoracic aneurysm's rupture, endovascular stent grafting is faster, less invasive, life-saving, and moreover, an ideal and unique alternative to open repair. Endovascular treatment of thoracic aortic aneurysms is achieved by transluminal placement of one or more stent-graft devices in the aorta which bridge the aneurysmal sac. This position excludes it from high-pressure aortic blood flow, allowing the development of thrombosis around the stent graft. However, this approach is subject to anatomic and logistic limitations, including appropriate aortic diameter, characteristics and extent of the aneurysm, proximal and distal landing zones, difficult peripheral arterial access, and the need for a wide range of stent graft sizes to be available for emergency use [Conrad 2010].

\section{CASE REPORT}

The patient in this case report is a 72-year-old man referred to our center from a regional hospital after the onset of acute and severe left chest and back pain. The patient was hypertensive, a heavy smoker, and had a severe atherosclerotic peripheral disease. In addition, he had severe claudication for more than one year and had no known history of an aortic aneurysm or coronary artery disease.

On admission, the patient was awake and responsive. $\mathrm{He}$ presented with dyspnea, transient hypotension, and left sided chest and back pain. He was transferred to ICU stable, and his transient hypotension was treated with intravenous fluid administration. Laboratory test results displayed hemoglobin at $9.8 \mathrm{~g} / \mathrm{dL}$, and creatinine at $0.67 \mathrm{mg} / \mathrm{dL}$. Physical examination 


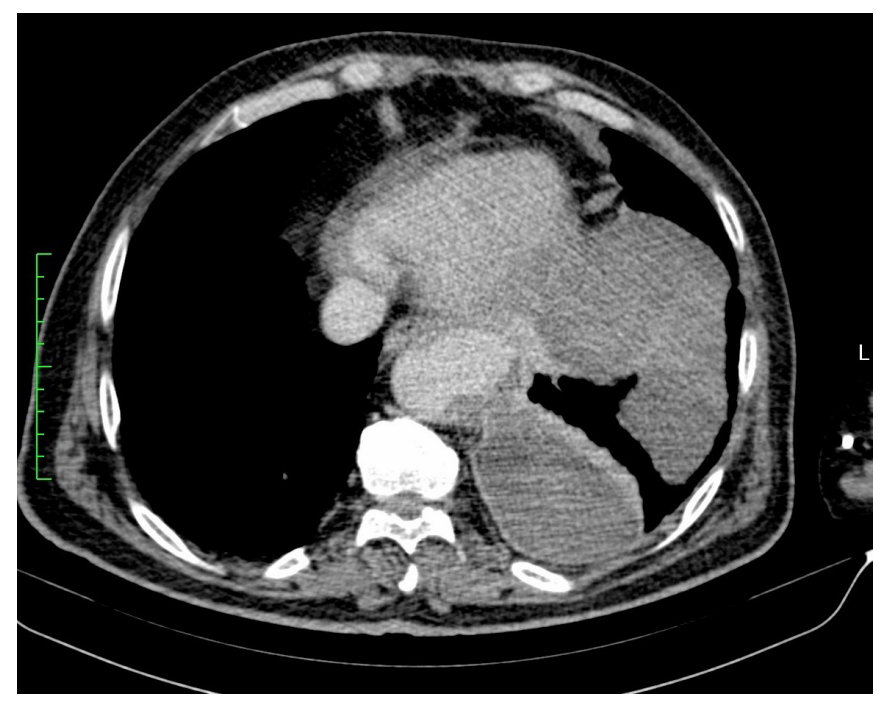

Figure 1. CTA showing the descending aortic aneurysm communicating with the left pleura..

revealed the absence of a murmur on the left hemithorax and weak bilateral femoral arterial pulse. X-ray revealed full opacity on the left hemithorax. Transthoracic echocardiography found normal left ventricular function without any valvular pathology. Computed tomography angiography (CTA) performed at the referring center described a $7.8 \mathrm{~cm}$ diameter, $6.8 \mathrm{~cm}$ long aneurysm, and a large amount of blood in the

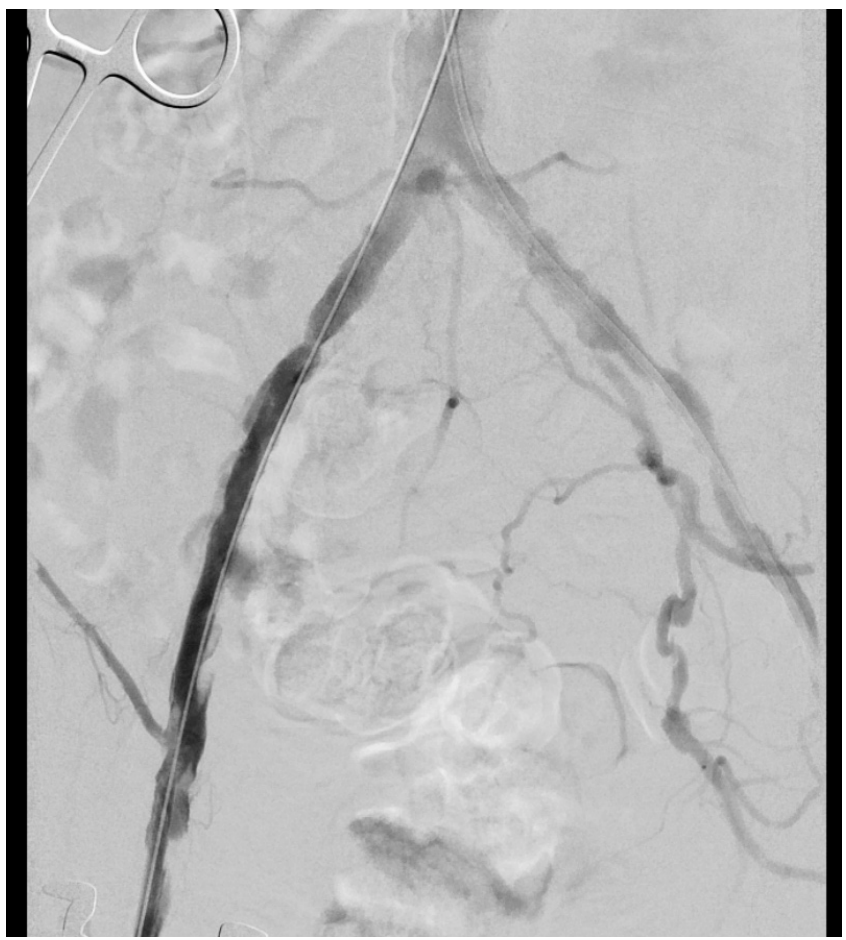

Figure 2. Arteriography showing severe calcifications of both iliac arteries and dissection of the left iliac artery.

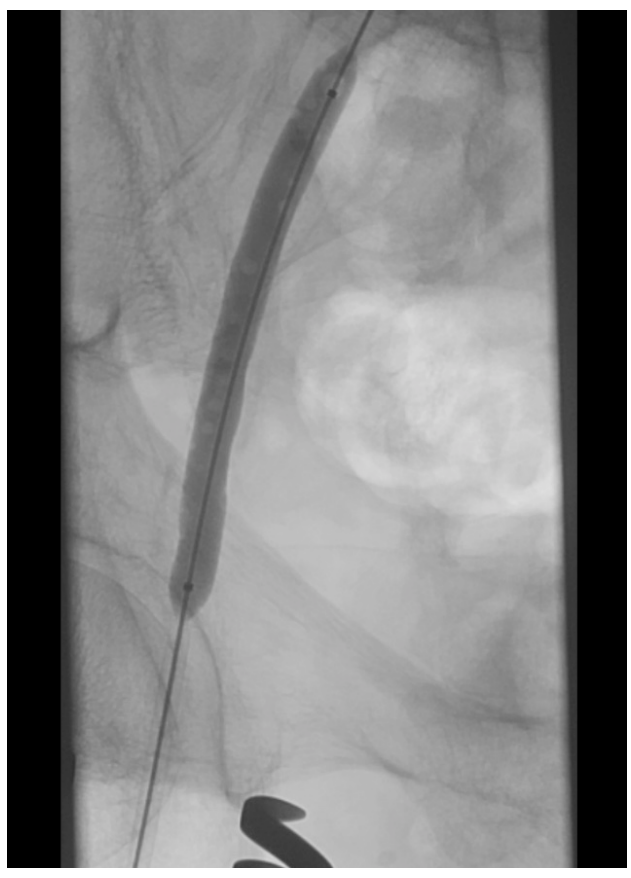

Figure 3. Right iliac artery dilatation..

left pleural cavity (Figure 1). The aneurysm was located in the distal third of the thoracic aorta, and showed signs of rupture. In addition, severe calcifications were discovered on both iliac arteries, and the remaining circulated lumen of both iliac arteries was found on CTA to be less than 2 to $3 \mathrm{~mm}$.

Emergency open surgery was considered to have extremely high risks, mainly because of the patient's associated pathologies. Because of this, our first choice was emergency TEVAR, which had lower risks. However, it was possible that it would be compromised by the patient's chronic peripheral arteriopathy. Preoperative cerebrospinal fluid (CSF) drainage was performed. Surgical access to the right femoral artery was performed under general anesthesia and an 8 Fr sheath was placed in the artery under direct vision. The contralateral femoral artery was used for angiographic control. Systemic heparin was administered. We initially performed a control arteriography which showed severe calcifications of both iliac arteries (Figure 2), and a dissection of the right internal iliac artery extended till the right inguinal ligament, most likely due to primary insertion of the guideline. We decided to dilate both iliac arteries with Abbott Vascular Armada 35 balloons, sizes $8.0 \mathrm{~mm}$ x $60 \mathrm{~mm}$ x $135 \mathrm{~mm}$ and $7.0 \mathrm{~mm}$ x 80 $\mathrm{mm} \times 135 \mathrm{~mm}$ (Figure 3), with very good results on the left iliac artery and partial stabilization of the dissection on the right iliac artery. This allowed us to continue the endovascular procedure.

A floppy wire was guided under fluoroscopy into the aortic arch. When in the proper position, the wire was exchanged with a soft catheter, and a stiffer wire was inserted through the catheter for device placement. The first inserted sheath was then exchanged with the appropriate device sheath. A stiff guidewire was advanced through the right external iliac artery 


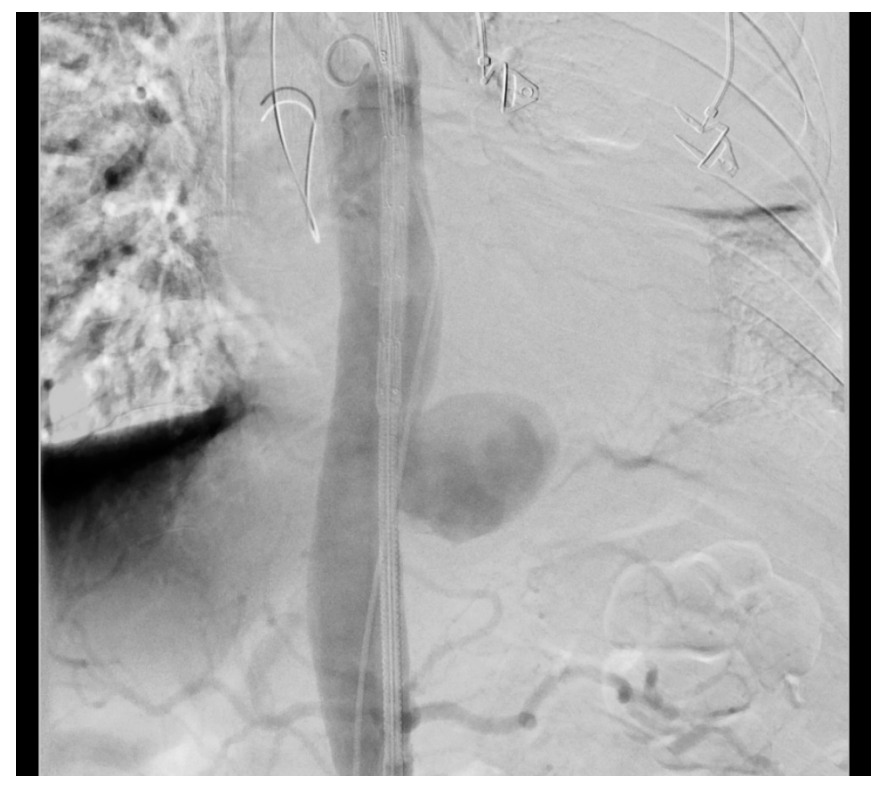

Figure 4. Intraoperative aortography with evidence of the descending thoracic aortic aneurysm.

into the ascending aorta. A new intraoperative aortography was obtained (Figure 4), which showed the aneurysm located in the distal third of the thoracic aorta. Under fluoroscopic guidance, a $36 \mathrm{~mm}$ by $20 \mathrm{~cm}$ long thoracic endoprosthesis (The Valiant Thoracic Stent Graft with the Captivia Delivery System), was loaded, positioned and deployed in the descending thoracic aorta covering the aneurysm. Both the proximal and distal landing zones were expanded with a Reliant stent graft balloon catheter. An angiography was performed in order to check for endoleaks and to verify if any additional stents were required. The complete angiography demonstrated total exclusion of the descending thoracic aortic aneurysm, correct position on the endograft with no evidence of any endoleaks (Figure 5), and a minimal residual dissection on the entire right iliac artery. We decided that no additional stents were necessary in order to stabilize this dissection.

Six hours later, before the patient was extubated, a chest tube was inserted to evacuate $1000 \mathrm{~mL}$ of blood from the left thorax cavity. Post-procedure, the patient's neurological evolution was good: there were no findings to indicate neurological complications, such as paralysis of the extremities. After 24 hours, the patient presented right inferior limb ischemia. We considered the possibility that the limb ischemia was due to the progression of the residual dissection on the right iliac artery, and emergency surgery was decided. First, we performed a thromboembolectomy with a Fogarty probe in order to eliminate any possible migrated clots from the TEVAR procedure. We did not extract any thrombotic or embolic material from the iliac or the femoral artery, but the arterial anterograde blood flow was considered as inadequate, and we performed an extra-anatomic axillofemoral bypass with a no. 8 Gore-Tex prosthesis. The postoperative evolution was clinically uneventful, and the patient was discharged from the hospital on the ninth day after TEVAR.

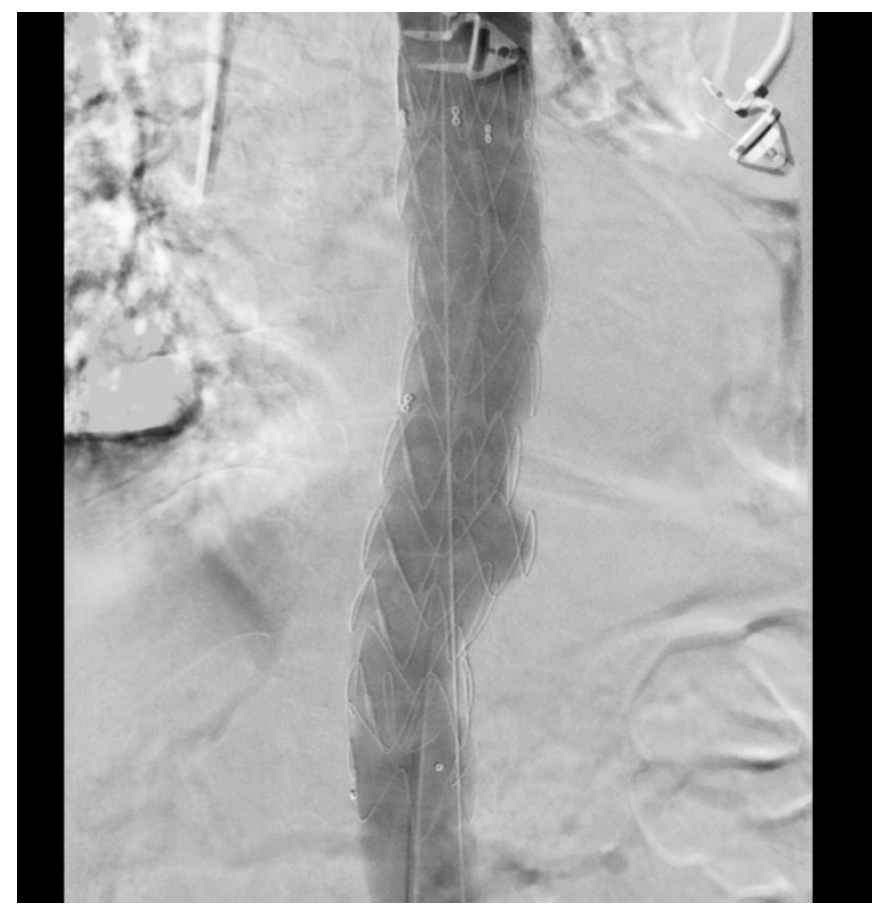

Figure 5. Postoperative arteriography showing correctly positioned stent graft.

CTA controls at one and three months after the procedure revealed a good evolution, with thrombosis around the stent graft and no evidence of aneurysm expansion or endoleaks.

\section{DISCUSSION}

In our patient, the CTA diagnosed the thoracic aortic aneurysm but did not show the precise location of the thoracic aortic rupture with active bleeding. The diagnosis of ruptured descending thoracic aortic aneurism (rDTAA) was suggested by the combination of hemothorax, hypovolemic shock, and thoracic aortic aneurysm. The severity of the peripheral arterial disease was the only unknown factor. Despite this, our decision for TEVAR was based on the patient's hemodynamic instability and the need for immediate intervention. Primary technical success was defined as complete exclusion of the aneurysm, stent graft patency, and restoration of blood flow immediately after TEVAR.

For the diagnosis of rDTAA, TEVAR is a minimally invasive approach and is an ideal alternative to open surgical repair for patients with high risk. TEVAR is now safe and effective in aneurysm exclusion and is associated with shorter operating times, a shorter need for mechanical respiratory support and a shorter stay in the ICU [Minami 2015; Botsios 2014]. Open surgical repair is a more invasive procedure than TEVAR, and patients are more likely to present with severe complications such as acute myocardial infarction, stroke, and paraplegia during or shortly after the operation [Johansson 1995]. Thoracotomy, aortic cross-clamping, cardiopulmonary bypass, and single-lung ventilation are all avoided 
with TEVAR [Minami 2015]. These advantageous characteristics of endovascular management of rDTAA appear to result in superior short-term outcomes compared with open repair, as was shown in different study groups which had periprocedural stroke rates of $0 \%$ to $7 \%$ [Botsios 2014; Fattori 2003], and incidence rates for paraplegia between $0 \%$ and 5\% [Fattori 2003; Cambria 2002; White 2001; Dake 1998]. After open repair, the incidence of paraplegia was $8.7 \%$ [Jonker 2011]. Immediate awakening of the patient, short hypotension during endograft positioning, appropriate fluid balance, maintenance of a mean intra-aortic pressure between 90 and $110 \mathrm{mmHg}$ after stent deployment, a central venous pressure below $10 \mathrm{mmHg}$, and a cerebrospinal fluid pressure management, are all factors that lead to a successful intervention [Hagendoorn 2014; Ullery 2011; Bavaria 2007; Chiesa 2005].

In recent years, an improvement is noted as a reduction in mortality rates for patients treated with TEVAR for rDTAA compared to open surgical repair. In our patient, left hemothorax was associated with rDTAA. Hemothorax may provoke compression of the esophagus and the cardiovascular structures of the thorax, and blood clots may adhere to the lung and pleura, making them difficult to remove with a single chest tube. This could lead to respiratory insufficiency, and later, even to thoracic infection [Bradley 2013; Boersma 2010]. Hemothorax evacuation with a chest drainage tube instead of a thoracotomy was possible due to the absence of organized blood clots.

Piffaretti concluded that in patients with modified preoperative respiratory parameters, prompt hemothorax evacuation reduces postoperative mortality [Piffaretti 2015]. All the anatomic considerations such as aortic diameter, characteristics and extent of the aneurysm, and proximal and distal landing zones, should be taken into consideration. The proximal landing zone is under the left subclavian artery and requires a proximal length of $2 \mathrm{~cm}$ prior to the aneurysm, and the aortic inner diameter must be between $23 \mathrm{~mm}$ and $37 \mathrm{~mm}$. The distal landing zone should be at $2 \mathrm{~cm}$ above the celiac trunk, and requires the same inner diameter as the proximal landing zone. In the presented case, we evaluated all these parameters on the CTA examination and the patient was considered eligible for the TEVAR procedure.

For implantation, it is required to have appropriate femo$\mathrm{ral}$ and iliac arteries sizes (more than $8 \mathrm{~mm}$ diameter), lack of tortuosity, and no atherosclerotic diseases. The difficulty of the presented case was the presence of peripheral arterial disease. Without primary dilatation of the iliac artery, it would have been impossible to advance the device with the thoracic endoprosthesis to the aneurysm. This specific category of patients, with evidences of severe peripheral arterial disease, are more likely to develop post-procedural complications such as dissection of an atherosclerotic artery or thromboembolic events. Unfortunately, performing only arterial dilatation in severe atherosclerotic dissected artery may be not enough to maintain the patency of the iliac artery. If it is possible, stabilizing post-procedural dilatation with stents can be a good solution.

Postoperative endoleaks must be prevented with careful CTA analysis of the thoracic aorta, precise preoperative stent-graft planning, and proper endograft deployment [Jonker 2011]. In our patient, angiography showed successful deployment and functional exclusion of the aneurysm, and the CTA at one month after the procedure showed thrombosis in the remaining aneurysmal cavity after TEVAR, with no evidence of endoleaks. The rate of endoleak immediately after thoracic aortic aneurysm stent-graft placement has been reported to be between $4 \%$ and $24 \%$ [Bortone 2004, Melnitchouk 2004]. Jonker reported a significantly lower 30-day mortality in patients treated with TEVAR for rDTAA compared to open surgical repair $(18.9 \%$ versus $33.3 \%$, p $=0.016$, respectively) [Jonker 2010]. In terms of morbidity when comparing open surgical repair with TEVAR, they reported a significantly higher rate of pulmonary complications ( 31.9 vs. $17.4 \% ; \mathrm{p}=0.032$, respectively), acute renal failure (24.6 vs. $8.7 \%$; $\mathrm{p}=0.006$, respectively) and median length of hospital stay for surviving patients ( 22 days vs. 8 days; $\mathrm{p}<0.001$, respectively) [Jonker 2011]. Ishida reported patient survival rates at one, three, and five years were $90.9 \%, 85.4 \%$, and $77.5 \%$, respectively [Ishida 2004]. The presented case seems to offer the best solution in high-risk patients with rDTAA. It may be the best evidence that this is an available treatment option regarding the management of this life-threatening disease, even if is associated with a severe peripheral arterial disease.

\section{CONCLUSIONS}

Emergency treatment of rDTAA is challenging in patients with severe atherosclerotic peripheral disease due to technical difficulties regarding the insertion of the endoprosthesis. Arterial balloon dilatation of the iliac arteries and CTA reconstructions are always helpful. Endovascular repair in patients with rDTAA can be a strong alternative to open surgery, and in addition, some extra-anatomic revascularization procedure may evolve as a superior treatment method for patients with appropriate indications.

\section{ACKNOWLEDGMENT}

The authors express their gratitude to Denisa Muraru, $\mathrm{MD}, \mathrm{PhD}$, for her encouragement and helpful advice.

\section{REFERENCES}

Bavaria JE, Appoo JJ, Makaroun MS, et al. 2007. Endovascular stent grafting versus open surgical repair of descending thoracic aortic aneurysms in low-risk patients: a multicenter comparative trial. J Thorac Cardiovasc Surg 133:369-77.

Boersma WG, Stigt JA, Smit HJ. 2010. Treatment of haemothorax. Respir Med 104:1583-7.

Bortone AS, De CE, D'Agostino D, et al. 2004. Endovascular treatment of thoracic aortic disease: four years of experience. Circulation 110:II-262-7.

Botsios S, Frömke J, Walterbusch G, et al. 2014. Endovascular treatment for nontraumatic rupture of the descending thoracic aorta: long-term results. J Card Surg 29:353-8. 
Bradley M, Okoye O, DuBose J, et al. 2013. Risk factors for post-traumatic pneumonia in patients with retained haemothorax: results of a prospective, observational AAST study. Injury 44:1159-64.

Cambria RP, Brewster DC, Lauterbach SR, et al. 2002. Evolving experience with thoracic aortic stent-graft repair. J Vasc Surg 35:1129-36.

Chiesa R, Melissano G, Marrocco-Trischitta MM, et al. 2005. Spinal cord ischemia after elective stent-graft repair of the thoracic aorta. J Vasc Surg 42:11-7.

Choi JS, Oh SJ, Sung YW, et al. 2016. Early experiences with the endovascular repair of ruptured descending thoracic aortic aneurysm. Korean J Thorac Cardiovasc Surg 49:73-9.

Conrad MF, Ergul EA, Patel VI, et al. 2010. Management of diseases of the descending thoracic aorta in the endovascular era: a medicare population study. Ann Surg 252:603-10.

Dake MD, Miller DC, Mitchell RS, et al. 1998. The "first generation" of endovascular stent-grafts for patients with aneurysms of the descending thoracic aorta. J Thorac Cardiovasc Surg 116:689-703.

Fattori R, Napoli G, Lovato L, et al. 2003. Descending thoracic aortic diseases: stent-graft repair. Radiology 229:176-83.

Hager A, Kaemmerer H, Rapp-Bernhardt U, et al. 2002. Diameters of the thoracic aorta throughout life as measured with helical computed tomography. J Thorac Cardiovasc Surg 123:1060-66.

Hogendoorn W, Schlösser FJ, Muhs BE, et al. 2014. Surgical and anaesthetic considerations for the endovascular treatment of ruptured descending thoracic aortic aneurysms. Curr Opin Anaesthesiol 27:12-20.

Ishida M, Kato N, Hirano T, et al. 2004. Endovascular stent-graft treatment for thoracic aortic aneurysms: short to midterm results. J Vasc Interv Radiol 15:361-7.
Johansson G, Markstrom U, Swedenborg J. 1995. Ruptured thoracic aortic aneurysms: a study of incidence and mortality rates. J Vasc Surg 21:985-8.

Jonker FH, Verhagen HJ, Lin PH, et al. 2011. Open surgery versus endovascular repair of ruptured thoracic aortic aneurysms. J Vasc Surg 53:1210-6.

Jonker FH, Trimarchi S, Verhagen HJ, et al. 2010. Meta-analysis of open versus endovascular repair for ruptured descending thoracic aortic aneurysm. J Vasc Surg 51:1026-32.

Lam CR, Aram HH. 1951. Resection of the descending thoracic aorta for aneurysm; a report of the use of a homograft in a case and an experimental study. Ann Surg 134:743-52.

Melnitchouk S, Pfammatter T, Kadner A, et al. 2004. Emergency stentgraft placement for hemorrhage control in acute thoracic aortic rupture. Eur J Cardiothorac Surg 25:1032-8.

Minami T, Imoto K, Uchida K, et al. 2015. Thoracic endovascular aortic repair for ruptured descending thoracic aortic aneurysm. J Card Surg 30:163-9.

Piffaretti G, Menegolo M, Kahlberg A, et al. 2015. Hemothorax management after endovascular treatment for thoracic aortic rupture. Eur J Vasc Endovasc Surg 50:608-13.

Ullery BW, Wang GJ, Low D, et al. 2011. Neurological complications of thoracic endovascular aortic repair. Semin Cardiothorac Vasc Anesth 15:123-40.

White RA, Donayre CE, Walot I, et al. 2001. Endovascular exclusion of descending thoracic aortic aneurysms and chronic dissections: initial clinical results with the AneuRx device. J Vasc Surg 33:927-34. 